\title{
Multiscale approach for magnetization dynamics: unraveling exotic magnetic states of matter
}

\author{
É. Méndez, ${ }^{1}$ M. Poluektov, ${ }^{2}$ G. Kreiss, ${ }^{3}$ O. Eriksson,,${ }^{1,4}$ and M. Pereiro ${ }^{1}$ \\ ${ }^{1}$ Division of Materials Theory, Department of Physics and Astronomy, Uppsala University, Box 516, 75121 Uppsala, Sweden \\ ${ }^{2}$ International Institute for Nanocomposites Manufacturing, WMG, University of Warwick, Coventry CV4 7AL, United Kingdom \\ ${ }^{3}$ Department of Information Technology, Uppsala University, Box 337, SE-751 05 Uppsala, Sweden \\ ${ }^{4}$ School of Science and Technology, Örebro University, SE-70182 Örebro, Sweden
}

(Received 21 August 2019; revised manuscript received 5 November 2019; published 28 January 2020)

\begin{abstract}
Crystallographic lattice defects strongly influence dynamical properties of magnetic materials at both microscopic and macroscopic length scales. A multiscale approach to magnetization dynamics, which is presented in this paper, accurately captures such effects. The method is illustrated using examples of systems with localized, nontrivial topological properties, e.g., in the form of skyrmions and domain walls that interact with lattice dislocations. Technical aspects of the methodology involve multiscale magnetization dynamics that connect atomistic and continuum descriptions. The technique is capable of solving the Landau-Lifshitz-Gilbert equations efficiently in two regions of a magnetic material—-the mesoscopic and the atomistic regions, which are coupled in a seamless way. It is demonstrated that this methodology allows simulating realistically sized magnetic skyrmions interacting with material defects and complex physical effects.
\end{abstract}

DOI: 10.1103/PhysRevResearch.2.013092

\section{INTRODUCTION}

It is of paramount importance in material science to simulate the behavior of matter both at small and large length scales. New and exciting macroscopic phenomena in materials are usually driven by the perturbation of physical properties at local and very small length scales. For example, dislocations or defects of atomic positions influence plastic deformation of materials and in magnetic media, they are a source of domain-wall pinning and the Barkhausen effect. In many cases, local properties cannot be disentangled from the global properties of the material, in which they are embedded, and, consequently, both spatial scales are required to be treated on equal footing. This is a highly nontrivial task, which this paper addresses. Technological applications, e.g., the promising area of spintronics [1] and the evolving field of quantum computing [2], bring relevance to the field of magnetism. Scientific advancements in this area are provided by several experimental techniques, e.g., pump-probe measurements of magnetism [3]. For interpretation of experiments, simulations are highly relevant and allow understanding or even predict interesting phenomena. In essentially all fields of physics, simulation tools are used in parallel to experimental observations.

Magnetic phenomena can be studied at a variety of scales, from macroscopic to atomic. Different models are traditionally used at each level in order to consider specific physical phenomena that are significant for a given time and length scale. Two commonly used paradigms are the micromag-

Published by the American Physical Society under the terms of the Creative Commons Attribution 4.0 International license. Further distribution of this work must maintain attribution to the author(s) and the published article's title, journal citation, and DOI. netic model and the atomic description that is based on the Heisenberg Hamiltonian. Micromagnetic theory [4] describes phenomena typically at the micrometer length scale. This methodology is well established and has been used for several decades. It is known to provide results of a relatively high degree of agreement with experimental data, at least for the length scale and time range it is designed for. Fundamental to this model is the assumption that the magnetization varies smoothly in space and microstructural information is embedded into effective parameters. These conditions impose a limit on the applicability of this model to small magnetic structures or intricate atomistic arrangements. On the other hand, the Heisenberg spin model provides a discrete, atomic-scale description of magnetism. This model is capable of representing magnetization dynamics on an atomic length scale, naturally including effects of lattice irregularities [5]. However, the computational cost of atomistic spin dynamics simulations makes it unfeasible for large computational domains. Therefore, it has difficulties describing the properties of samples at sizes that are used in some experimental measurements.

Micromagnetic models allow distributing computational points in a controlled manner. These methods have been reported in the literature and are based on finite differences, e.g., in Refs. [6,7], or finite elements, e.g., in Ref. [8]. The atomistic model [9-12] does not permit redistributing computational points, but it can be used in combination with micromagnetic models leading to various multiscale schemes. There are several versions of such coupling schemes developed in application to magnetic materials [13-16] and the reader is referred to a review article published in Ref. [17] for an exhaustive overview.

The aim of this paper is to demonstrate the applicability of a multiscale method to analyze complex magnetization dynamics in systems where large and small length scales play a role, e.g., skyrmion dynamics and its influence from 
defects on the atomic scale. Limitations of this methodology are established by considering a set of examples that are challenging for any similar numerical method that aims at simulating magnetization dynamics of materials. To perform the investigation, an implementation of a multiscale method has been made in the UPPASD software [18,19], taking onboard techniques from Refs. [20,21]. The micromagnetic model is shown here to have sufficient accuracy for regions where the magnetization varies smoothly, while atomic-level simulations can reproduce the much more complex behavior at shorter length scales. Framing a small atomistic region with a thick micromagnetic layer in a way proposed in this paper permits estimating how perturbations propagate in the material, while avoiding reflections that pollute the region of interest and keeping the computational effort and accuracy mainly within the atomistic part. As is demonstrated below, the multiscale method outlined here is able to simulate dynamics of topological excitations, e.g., skyrmions with realistic (experimental) size, and their interaction with atomistic defects, such as dislocations.

\section{METHODS}

The type of atomistic-continuum coupling considered in this paper is the partitioned-domain method. In this scheme, the computational domain is split into regions that belong to different models, with an explicit interface between the regions. There are two conceptually distinct atomisticcontinuum coupling methodologies: the energy-based and the force-based coupling [22]. There are two major challenges for partitioned-domain atomistic-continuum coupling methods: handling nonlocal atomistic interactions, which is relevant for the energy-based methods applied in both statics and dynamics, and averaging high-frequency excitations within the atomistic solution that must not reflect from the boundary between the atomistic and the coarsely discretized continuum regions. It must be emphasized that the latter is not an issue of coupling methods and results from disparate discretizations of the regions. Atomistic-continuum coupling methods originate from the field of mechanics and date back to the 1990s and, therefore, the highlighted issues were largely addressed in application to modeling deformation; e.g., Ref. [23] solves the local/nonlocal coupling issue in the energy-based methods in mechanics and Ref. [24] proposes a way of dealing with highfrequency atomistic excitations. In the field of magnetism, the local/nonlocal coupling has been addressed in Ref. [20], while a technique for averaging high-frequency spin motion at the interface has been proposed in Ref. [21].

The technical aspects of the method proposed and used here involves the merging of an atomistic and a continuum description of the magnetization dynamics into an established software: UPPASD [19]. Most of the technical aspects used in this work have been introduced previously [5,18,20,21] and, therefore, relevant technical aspects of this work are presented in the Supplemental Material [25] (see note S1).

\section{RESULTS}

It is well known that it is not possible to construct a completely error-free atomistic-continuum coupling, as dis- cussed in Ref. [22]. Previous studies [20,21] focused on analyzing the errors introduced by the coupling approach both analytically and computationally. The aim of the examples of this paper is to show that it is possible to construct the coupling such that atomistic and continuum regions behave in a similar way and the errors introduced by the atomisticcontinuum interface are sufficiently small and do not influence the microscopic and the macroscopic behaviors. In the general case, the atomistic-continuum coupling errors depend on the continuum discretization and on the relation of magnetization gradient to atomistic lattice spacing [20].

In the computational examples and figures, Cartesian coordinates $x, y, z$ correspond to the horizontal, the vertical, and the out-of-plane axes, respectively. In the figures, the red and the blue colors indicate magnetization pointing in plane and out of plane, respectively.

\section{A. Domain-wall motion}

A basic domain-wall motion, with a Hamiltonian that involves Heisenberg exchange and uniaxial anisotropy, is considered. The simulations consider two regions of opposing magnetization, each having orientation parallel to the lowest energy direction, specified by the easy axis [26]. The precise material parameters for the simulations of domain-wall motion are detailed in the Supplemental Material (note S3a) [25]. In this example, the domain-wall width is approximately 50 interatomic distances. The simulation shown in Fig. 1(a) shows that the domain wall, which starts in the continuum region, is not modified or distorted when it reaches the atomistic region [white/yellow box in the center of Fig. 1(a)]. This illustrates that the handshake between the two regions is seamless and that an object, which is not expected to change its shape, correctly maintains its original configuration when moving across interfaces between the regions. The same conclusion is reached when considering the domain wall that is leaving the atomistic region and entering the continuum region. The dynamics of the domain wall is found in the Supplemental Material (video 1) [25].

\section{B. Domain-wall motion and the Barkhausen effect}

The cause of the Barkhausen effect has been discussed previously in Ref. [27]. Materials usually contain irregularities in the crystallographic lattice, such as dislocations, stacking faults, impurity atoms, vacancies, and lattice distortions due to microstresses. A domain wall typically needs a local increase in energy around the defect to cross one of these irregularities and, therefore, becomes pinned-parts of the domain wall near the defect lag behind the rest of the domain wall. The unpinning of the domain wall causes a detectable change in the magnetic flux, which was picked up in Barkhausen's coils.

The numerical examples investigated here consider defects in the form of microscopically small, regular shaped holes in the hexagonal crystal lattice. Snapshots for different times of the domain-wall motion are shown in Figs. 1(b)-1(f) and are seen to represent the wall moving over the defect. Regions of the domain wall that come close to the hole move quickly towards the hole and get stuck around it, as can be seen in Fig. 1(c). The domain wall is stuck for an appreciable time, 

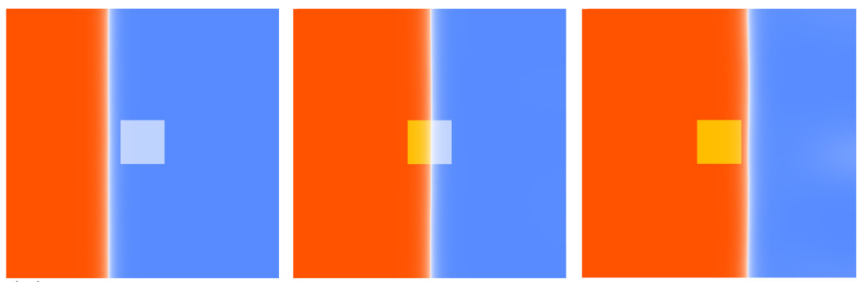

(a)
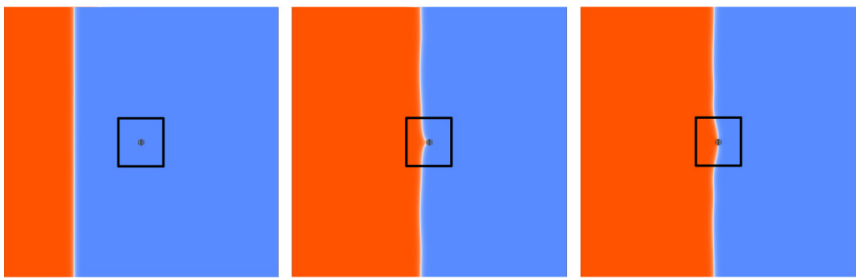

(b) $\mathrm{t}=500 \mathrm{ps}$

(c) $\mathrm{t}=1476 \mathrm{ps}$

(d) $\mathrm{t}=1540 \mathrm{ps}$
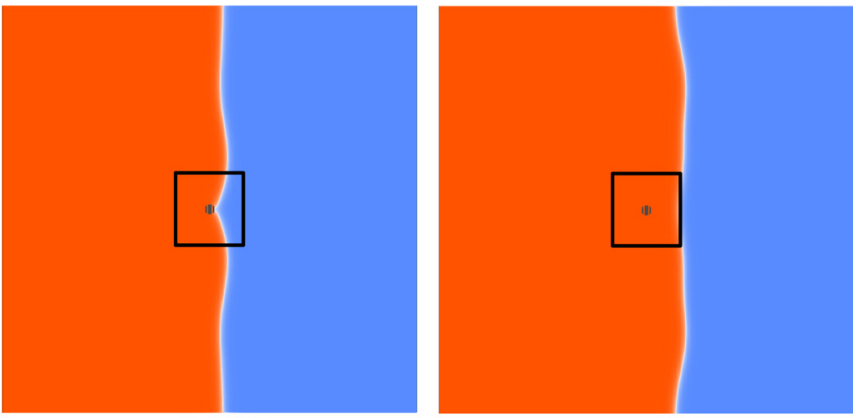

(e) $\mathrm{t}=1758 \mathrm{ps}$

(f) $\mathrm{t}=2000 \mathrm{ps}$

FIG. 1. (a) Domain-wall crossing atomistic region, which is indicated by the white/yellow square. (b)-(f) Snapshots at successive steps of the time evolution of the domain wall crossing an atomistic region containing a round hole, which is indicated by the black color. The atomistic region is indicated by the black frame.

even if the wall in the rest of the material keeps moving, as can be seen in Fig. 1(e). Eventually, in just a relatively short period of time $(\approx 200-500 \mathrm{ps})$ a substantial amount of the material changes its magnetization. In an experiment, such fast changes of the magnetization would lead to big changes in the magnetic flux that is connected to the Barkhausen effect (see the Supplemental Material, video 2 [25]). Although micromagnetic models have successfully discussed the Barkhausen effect [28] and generate results similar to those of Fig. 1, the approach adopted here has greater descriptive possibilities due to treatment of the defect region in an atomistic way, with unique properties of each atom around the defect and with parameters of the spin Hamiltonian that are evaluated from the first-principles theory.

\section{Topological magnetic states}

In condensed-matter physics, magnetic skyrmions are spatially localized excitations that preserve their shape. These skyrmions are homotopically distinct from the ferromagnetic state, meaning that there exists no continuous transformation that transforms the magnetic texture of a skyrmion to the ferromagnetic state. This topological argument has been discussed critically in the literature, particularly with respect to stability and the possibility of using these objects as carriers of information $[29,30]$. Skyrmions have been observed experimentally, both in static and dynamic conditions [31].

Magnetic skyrmions come in two versions-hedgehog skyrmions have their moments pointing in the radial direction, while vortex skyrmions have their moments circulating around the center. A particular type of skyrmion depends on the direction of the $\mathbf{D}_{i j}$ vector with respect to the vector $\mathbf{r}_{i j}$ connecting atomic positions $i$ and $j$. The material parameters used in the subsequent simulations, with the exception of skyrmion-dislocation interactions, are listed in the Supplemental Material, note S3b [25]. These parameters correspond to a one-atom-thick layer of iron resting atop a substrate of iridium. It has already been demonstrated experimentally that skyrmions exit in this system [32].

\section{Spin spiral and skyrmion lattice}

Considering only the Heisenberg exchange, energy is lowered for an atom and its neighbors when their atomic moments become parallel. In contrast, the energy associated to the Dzyaloshinskii-Moriya interaction can be minimized when the magnetic moments of neighboring atoms are noncollinear and the minimum energy is found when moments form an angle of $90^{\circ}$. In materials where both terms contribute significantly, a competition between interactions may introduce many different low-energy states. One family of states of these magnets is known as the spin spiral. For these systems, locally, the variation of spins becomes relatively small in one direction and relatively large in an orthogonal direction. There is a tendency for directions of minimal variation to align, creating long lines of aligned magnetization. In this example, the multiscale approach is used to describe this class of materials.

The time evolution of the magnetic moments achieving the spin spiral state is shown in Figs. 2(a)-2(c) and in the Supplemental Material, video 3 [25]. Initially, the system is thermalized by using an annealing process - the temperature is decreased gradually starting from $100 \mathrm{~K}$ until it reaches $0.1 \mathrm{~K}$. After the thermalization process, the system obtained a ferromagnetic state with all moments pointing along the $z$ direction (data not shown). Afterwards, the magnetic moments are allowed to evolve in time at $T=0.1 \mathrm{~K}$, until the system converges into a spin spiral state, as shown in Figs. 2(a)-2(c). The stripes of opposing magnetization are easy to identify by the $z$ component of the moments. If an external field of a sufficient magnitude is applied along the $z$ axis to the spin-spiral state (5 $\mathrm{T}$ in the example here), skyrmions may appear in the material, forming what is known as a skyrmion lattice; see the Supplemental Material, video 4 [25], and Figs. 2(d)-2(f). In simulations of both the skyrmion lattice and the spin spiral configuration, the border between the micromagnetic and atomistic regions is completely transparent. This implies that the interface between two regions does not introduce any spurious errors and the time evolution of the magnetization behaves according to expectations in both regions - the size and the shape of skyrmions is the same in both regions.

In the above simulations, the introduction of micromagnetic regions brings an improvement of computational efficiency, compared to purely atomistic simulations. To 


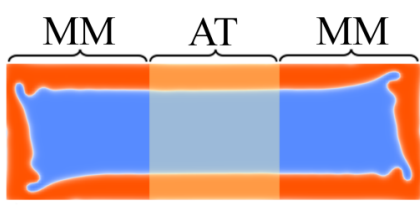

(a) $t=2$ ps

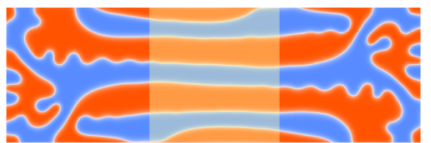

(b) $\mathrm{t}=6 \mathrm{ps}$

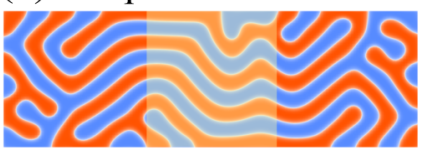

(c) $\mathrm{t}=80 \mathrm{ps}$

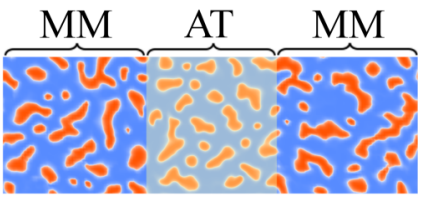

(d) $\mathrm{t}=2 \mathrm{ps}$

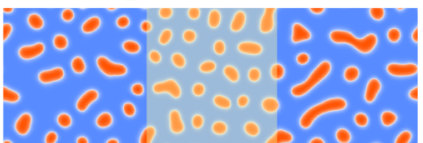

(e) $\mathrm{t}=8 \mathrm{ps}$

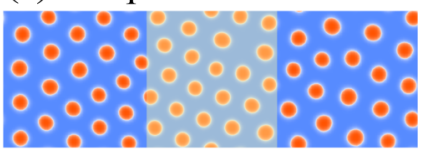

(f) $t=200 p s$

FIG. 2. (a)-(c) Three snapshots of the time evolution of the magnetization of one-atom-thick layer of Fe on Ir (111). The ferromagnetic initial state (not shown) evolves in time to a spin spiral configuration. (d)-(f) The application of an external magnetic field perpendicular to the plane of the sample drives the spin-spiral state shown in (c) to a skyrmion lattice state. Blue and red regions correspond to the magnetization pointing mainly along and opposite the $z$ direction, respectively. The sizes of the atomistic (AT) and the micromagnetic (MM) regions are indicated at the top of the figure.

characterize the coarseness of the system, an additional variable $c$ is introduced, which represents the number of atoms that fit between the continuum mesh nodes. Note that this parameter only specifies the coarseness of the simulation nodes of the continuum region. Hence, the value $c=1$ specifies the case when the atomistic and continuum regions have equal density of simulation atoms and simulation nodes, a case which represents essentially the computational effort of a purely atomistic simulation. In Fig. 3, the simulation time (CPU clock time measured in seconds after the simulation reaches 500 time steps) is shown for a two-dimensional (2D) ferromagnet with rectangular shape, in which both Heisenberg exchange and Dzyaloshinskii-Moriya (DM) interaction are significant, as a function of the number of simulation elements (atoms/nodes), for different values of $c$, i.e., for different

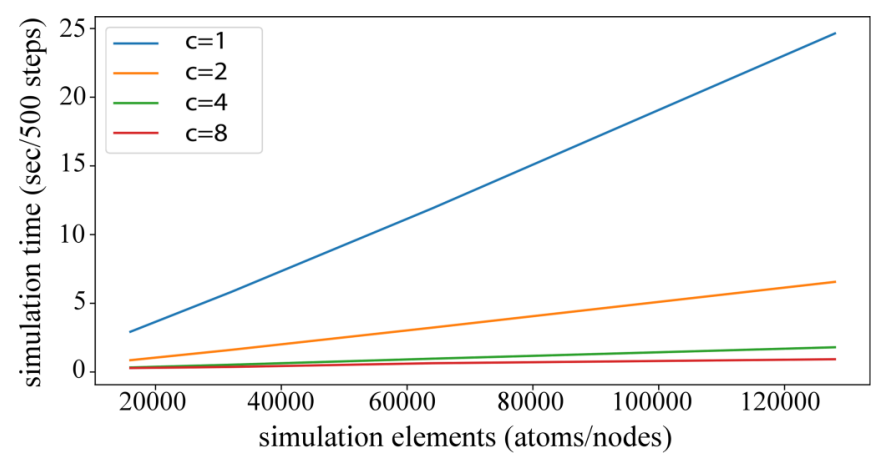

FIG. 3. The dependence of the simulation time on the number of the simulation elements (atoms and nodes) of a 2D ferromagnet with DM interaction on a square lattice. The parameter $c$ (coarseness) represents the number of atoms that fit between the continuum mesh nodes. The time step of $1 \mathrm{fs}$ is used. coarseness. The material parameters of this simulation are given in the Supplemental Material, note S3c [25]. The geometry considered in the simulations had rectangular-shaped regions of continuum and atomistic regions. In the simulation considered here, the initial magnetization points along the $z$ direction and since there is no external magnetic field, the magnetization evolves in time towards a spin-spiral state. The simulation time scales linearly with the size of the system for any choice of coarseness, as shown in Fig. 3. It may also be seen that the simulation time decreases significantly with increasing values of $c$. Naturally this comes at a cost, in terms of accuracy, and a suitable choice of $c$ must always be chosen with care, depending on the shape of the simulation cell and the dynamical response one is interested in. A closer inspection of the computational efficiency of the multiscale approach (data not shown) shows that the efficiency scales exponentially with $c$, clearly demonstrating the major advantage of the multiscale modeling approach suggested here, compared to a straight atomistic description.

\section{Single-skyrmion creation via microwave fields}

In this section, the creation process of skyrmions via an external magnetic field is illustrated, in a magnetic medium in which both the Heisenberg exchange and the DM interaction are important. Simulation details and materials parameters are given in the Supplemental Material, note S3d [25]. Initially, the magnetic moments point along the $z$ direction and are forced to align parallel by an external field of $6 \mathrm{~T}$. Single skyrmions are then stabilized by a local torque. Details are given in the Supplemental Material, note S5 [25]. The simulated time evolution of these skyrmions is shown in Fig. 4. The results from the micromagnetic model are shown in the topmost region, while the atomistic approach, on a hexagonal crystal lattice, is presented in the bottom region. The external field is applied to the entire material throughout the simulations, as it is found to keep the majority of magnetization aligned along the initial direction, which bounds the size of the skyrmion [31].

The skyrmions shown in Fig. 4 represent a metastable state. In absence of the external field, the system can lower its energy by expanding the radius of the skyrmion, while due to the external field, atomic moments favor alignment along the $z$ direction. The competition between the interactions results in a skyrmion with a size following a damped oscillatory mode. This behavior is known as the so-called "breathing mode." In Fig. 4 the inner magnetic texture of the skyrmions is shown, both for the atomistic and the micromagnetic regions. Due to the selected direction of the DM vector, the topological excitations in both regions are vortex skyrmions as shown in Fig. 4(e) and it can be seen that they have a similar shape and size [33]. The breathing mode is responsible for the alternating radius of the skyrmions depicted for various time steps in the simulations as shown in Figs. 4(b)-4(d). Since the resolution of the pulsed local field is constrained by the resolution of the underlying domain, slight differences in its effect are expected and are revealed at the first few steps of the simulation. Thus, right after the quenching of the magnetic pulse in Fig. 4, the waves of high frequency cause rapid changes in the spin direction of atoms in the region 


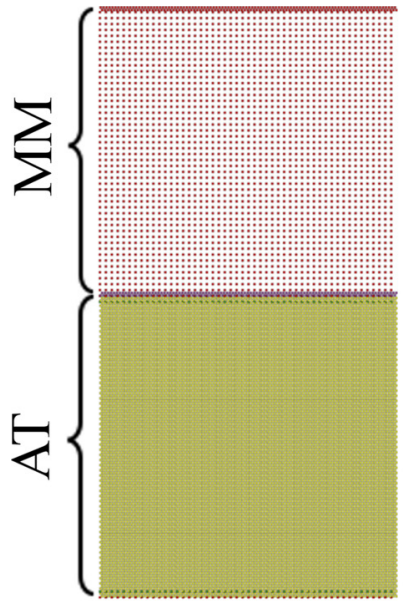

(a) Structure

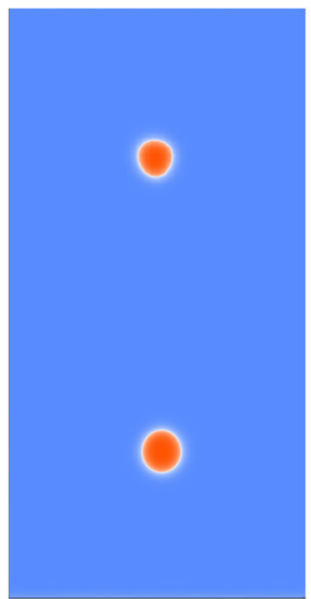

(b) $\mathrm{t}=10.3 \mathrm{ps}$

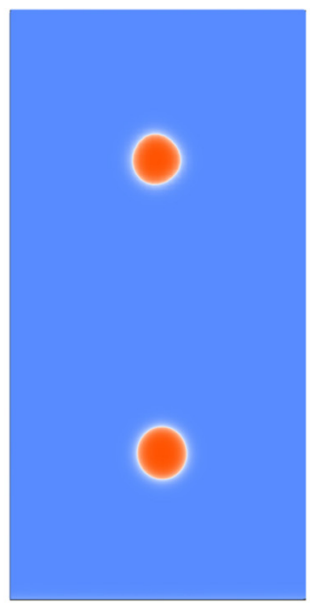

(c) $\mathrm{t}=11.2 \mathrm{ps}$

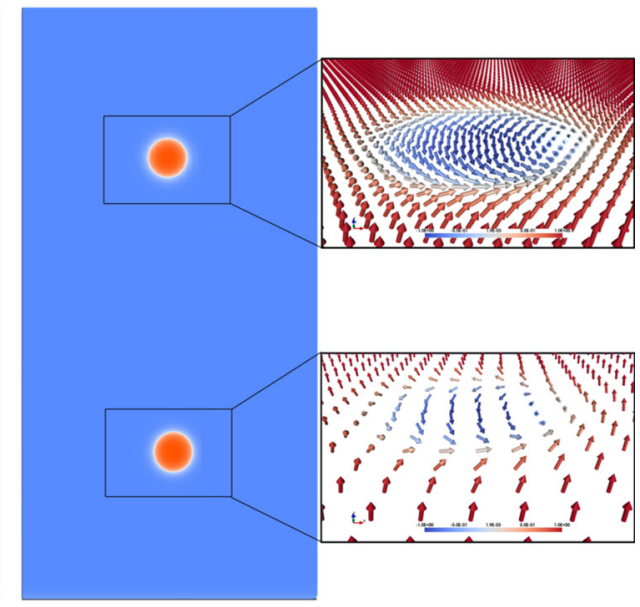

(d) $\mathrm{t}=40 \mathrm{ps}$

FIG. 4. Skyrmion introduced in the atomistic and the continuum regions of the same material. The size and the shape are shown as functions of time. The sizes of the atomistic (AT) and the micromagnetic (MM) regions are indicated on the left-hand side of the figure. In the right panels, the inner magnetic structures of the vortex skyrmions are shown. Notice that the distance between atoms and nodes is not scaled in the figure. In the simulation, the distance between atoms or nodes was taken to be 2.714 and $5.428 \AA$, respectively.

of the applied magnetic field. Single atoms in the atomistic region can change their spin as fast as physically allowed. However in the continuum, the magnetization of a cluster of atoms is represented by each node. It takes more energy and time to change the average magnetization of a group of atoms, so that the larger the group, the bigger the difference. As a consequence, nodes cannot oscillate so quickly and this effect can introduce a difference in the time evolution of the magnetization with respect to the atomistic one, only in cases as the one described in Fig. 4. Since a fundamental approximation in micromagnetics is based on the fact that the magnetization is being assumed to be a smooth function of the position, physical realizations where the magnetization varies rapidly in time can result in micromagnetic simulations to be less accurate than the corresponding atomistic simulations. This illustrates that a continuum description, even when tuned to have materials parameters selected to be as close as possible to represent the behavior of an atomistic region, does not capture the correct time evolution of the more accurate atomistic model. However, as the magnetic configuration evolves towards a stable stationary state, skyrmions of the continuum and atomistic descriptions become increasingly similar as shown in Fig. 4(d). The time evolution of both skyrmions is shown in the Supplemental Material, video 5 [25].

\section{Skyrmion-microstress interaction}

In this example, a smaller region of different magnetic anisotropy is considered, where locally residual microstresses in a material cause an enhanced anisotropy constant [34]. The simulation shown in Fig. 5 is set up similarly to that of Ref. [35], however, the triangular region with unique anisotropy (colored differently in the figure) is treated by an atomistic model on a hexagonal lattice. A hedgehog skyrmion is introduced (created as described in the previous section) and driven along the track by means of a spin current induced by STT. The material parameters for this example are given in the Supplemental Material, note S3e [25]. The direction of the spin current used in this simulation is given by the vector $(0.995,0.1,0)$. A small $y$ component is used to compensate the Magnus force that drives the skyrmion downwards as already reported in Ref. [36], resulting in motion along a straight line. A weak spin current of $4.5 \mathrm{~m} \mathrm{~s}^{-1}$ was found to be unable to push the skyrmion past the region of enhanced anisotropy. In this case, the skyrmion moves towards the irregularity, where it dissipates energy and angular momentum, which leads to the reduction in size, as shown in Figs. 5(a)-5(c) and the Supplemental Material, video 6 [25]. As a comparison, displayed in the right panels of Fig. 5 is a micromagnetic simulation with the same parameters as the ones used on the simulation of the left panels. In general, the physics keeps unchanged except by the fact that the skyrmion annihilates earlier in time (see the Supplemental Material, video 7 [25]). A strong spin current induced by the STT can push the skyrmion past the region of enhanced anisotropy-a value of $6 \mathrm{~m} \mathrm{~s}^{-1}$ is used for the simulation in Figs. 5(d)-5(f) and the Supplemental Material, video 8 [25] and was found to be strong enough to push the skyrmion past the microstress region. The micromagnetic simulation predicts again the same physics as in the multiscale method. The only difference is that the skyrmion shrinks substantially its size as it approaches the microstress region (see the Supplemental Material, video 9 [25]). Micromagnetic simulations overestimate a bit the anisotropy interaction in both weak and strong STT examples, and consequently, an atomistic spin dynamics method provides a more accurate description in the neighborhood of the microstress. An intermediate spin current of $5 \mathrm{~m} \mathrm{~s}^{-1}$ sustains the skyrmion as a stationary entity, even when it meets the magnetic irregularity (data not shown).

\section{Skyrmion-dislocation interaction}

An edge dislocation is a common crystallographic defect where one plane of atoms is missing a half, which distorts 


\section{Weak STT}

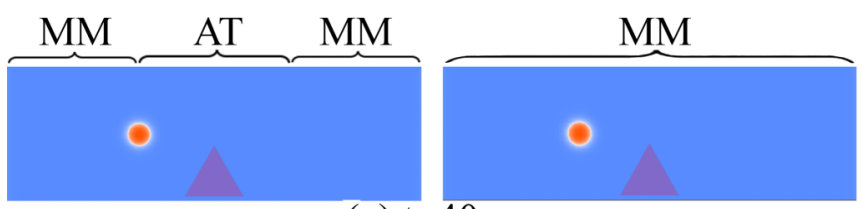

(a) $\mathrm{t}=40 \mathrm{ps}$

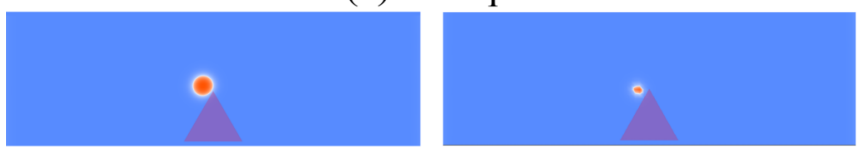

(b) $\mathrm{t}=80 \mathrm{ps}$

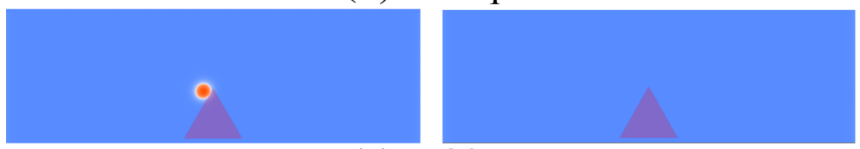

(c) $\mathrm{t}=90 \mathrm{ps}$

\section{Strong STT}

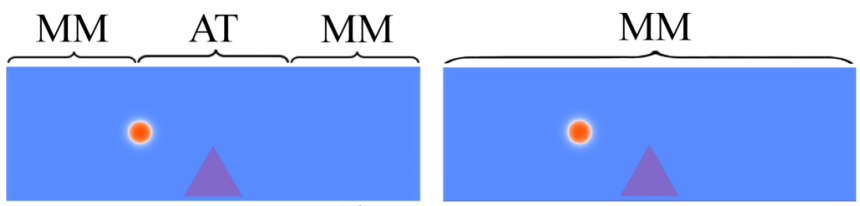

(d) $\mathrm{t}=30 \mathrm{ps}$

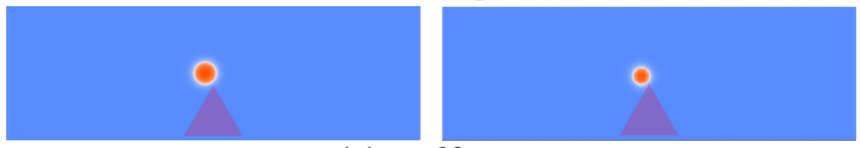

(e) $\mathrm{t}=60 \mathrm{ps}$

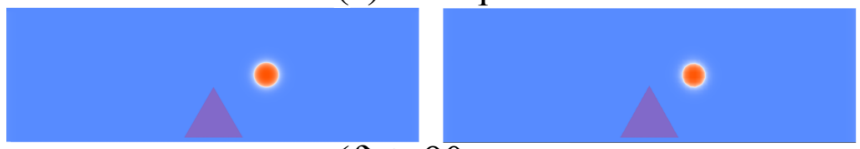

(f) $\mathrm{t}=90 \mathrm{ps}$

FIG. 5. Skyrmion interaction with the microstress-caused defect for two different values of the spin transfer torque (STT), after inducing a skyrmion via a microwave field. The triangular region marks the area where the magnetic anisotropy is larger than in the other regions. The sizes of the atomistic (AT) and the micromagnetic (MM) regions are indicated at the top of the figure.

nearby planes of atoms. Such defects are unavoidable in experimental samples, where they may influence the magnetic properties in a decisive way and in this subsection, the dislocation-affected skyrmion motion is illustrated. Results of this section cannot be obtained by a continuum model alone and illustrate the strength of the atomistic-continuum multiscale approach. The process of determining the positions of atoms within the dislocation is described in the Supplemental Material, note S6 [25]. Material parameters are given in the Supplemental Material, note S3f [25].

The sequence of images in Fig. 6 show snapshots of the skyrmion when it moves across the computational domain. Figures 6(a)-6(f) (Supplemental Material video 10 [25]) and 6(g)-6(l) (Supplemental Material video 11 [25]) correspond to the STT vectors with components $(1.25,0.175,0)$ and $(5,0.5,0) \mathrm{m} \mathrm{s}^{-1}$, respectively. In the latter case (i.e., relatively strong STT), the skyrmion interacts with the defect, however, it overcomes the region around the defect and subsequently

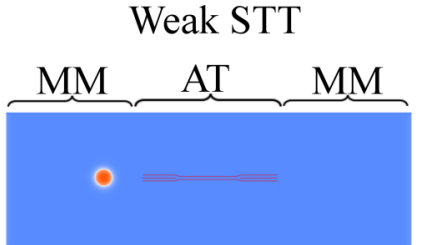

(a) $\mathrm{t}=250 \mathrm{ps}$

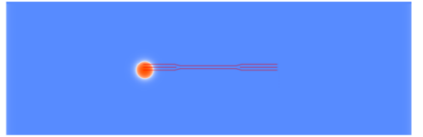

(b) $\mathrm{t}=600 \mathrm{ps}$

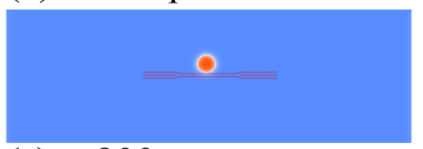

(c) $\mathrm{t}=900 \mathrm{ps}$

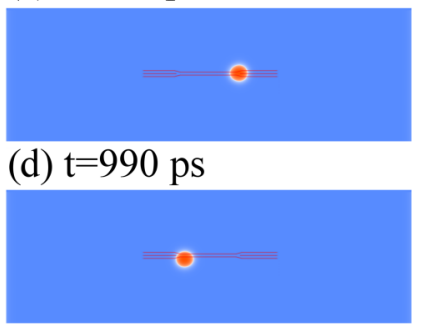

(e) $t=1300$ ps

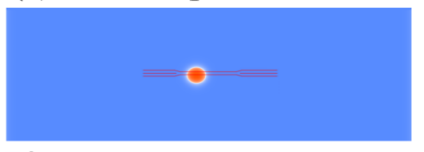

(f) $\mathrm{t}=1400 \mathrm{ps}$
Strong STT

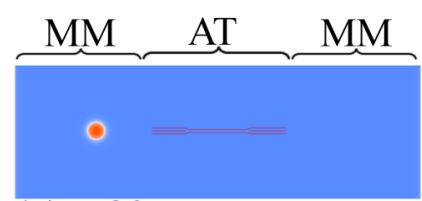

(g) $\mathrm{t}=30 \mathrm{ps}$

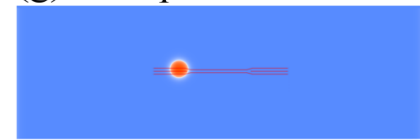

(h) $\mathrm{t}=200 \mathrm{ps}$

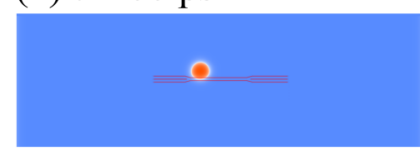

(i) $\mathrm{t}=230 \mathrm{ps}$

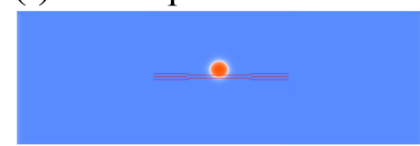

(j) $\mathrm{t}=254 \mathrm{ps}$

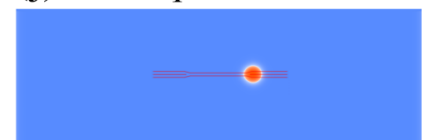

(k) $\mathrm{t}=300 \mathrm{ps}$

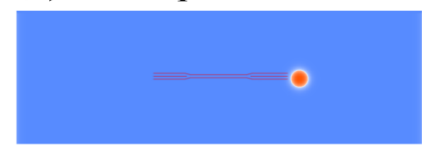

(l) $\mathrm{t}=400 \mathrm{ps}$
FIG. 6. Skyrmion-dislocation interaction, illustrated at different snapshots in time. The red lines represent atomic planes and visualize the dislocation. The brackets on the top of the figure indicate the atomistic (AT) and the micromagnetic (MM) regions. The dislocation is fully included in the atomistic region.

leaves the atomistic region, moving to the continuum. For a weaker STT current, the skyrmion moves at the top of the row of atoms of the dislocation. Once it comes to the end of the missing row of atoms, which is indicated by the red lines in Fig. 6, it moves to the bottom of the dislocation and changes direction-it moves backwards, against the direction of the STT. After reaching for the second time the left-hand side of the dislocation defect, the direction the skyrmion reverses again. Such oscillatory behavior of the skyrmion around the dislocation is repeated several times. Due to damping, the skyrmion travels a shorter path each iteration, until it is finally trapped by the defect as shown in Fig. 6(f). This counterintuitive behavior, where for a certain time the skyrmion moves against the direction favored by the STT, is impossible to observe within a continuum model.

In Fig. 7 and Supplemental Material video 12 [25], the time evolution of $x$ and $y$ components of the magnetization of the skyrmion is shown in the neighborhood of the dislocation. The colors indicate the direction of the major component of the magnetization in the $x y$ plane-red, yellow, green, and blue colors correspond to the directions of the magnetization primarily along right, down, left, and up directions, 


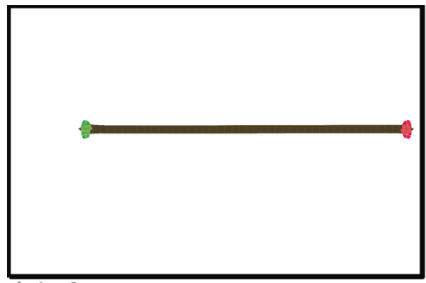

(a) $0 \mathrm{ps}$

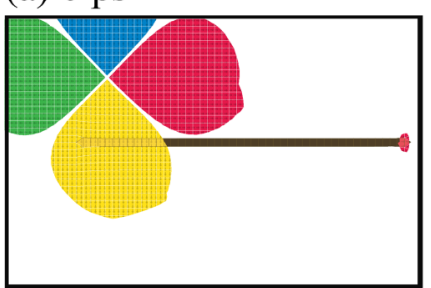

(c) $881 \mathrm{ps}$

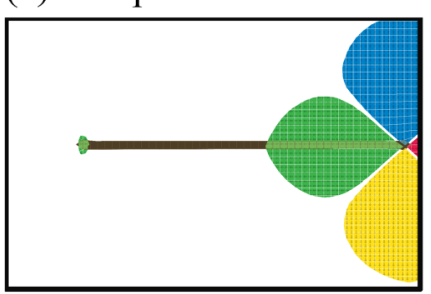

(e) $1034 \mathrm{ps}$

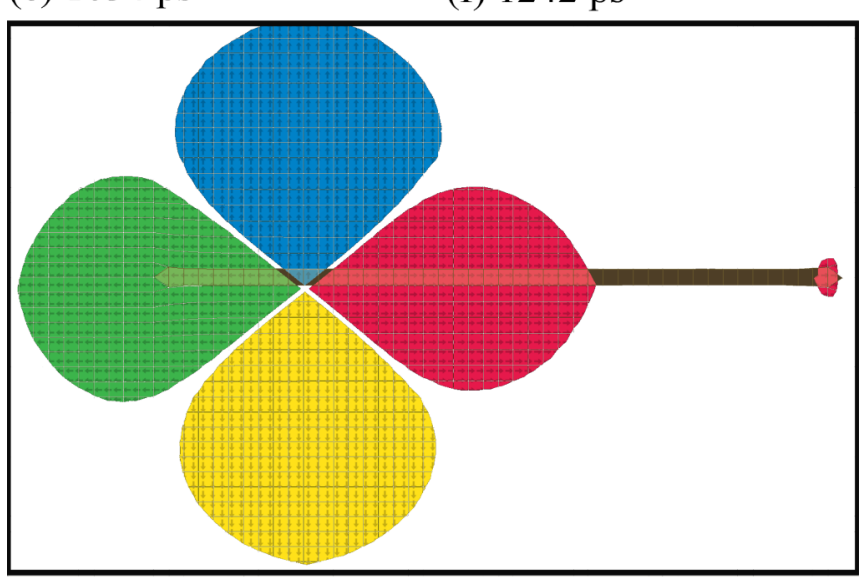

(g) $1759 \mathrm{ps}$

FIG. 7. Skyrmion-dislocation interaction, illustrated at different moments in time, for which only $x$ and $y$ components of the magnetization are shown. The simulation of Figs. 6(a)-6(f) is demonstrated. Red, yellow, green, and blue colors stand for the directions of the magnetization along right, down, left, and up directions, respectively. The brown/dark line visualizes the dislocation.

respectively. The same color pattern is used to describe the topology of the magnetic interactions at the end points of the dislocation. Since the DM vector is in plane and is perpendicular to the bond direction, the dislocation on the left end point introduces a local distortion of the DM interaction in such a way that it favors magnetization pointing opposite to the $x$ direction. For the right end point of the dislocation, the situation is reversed. As a consequence, the ground-state magnetic configuration is achieved when the atomic magnetic moments on the left and the right end points are tilted to the left and to the right, respectively, as shown in green and red colors in Fig. 7(a). The white background color indicates that the magnetization is pointing along the $z$ direction.

As depicted in Fig. 7, the skyrmion stays on the upper side of the defect when moving to the right and in the lower side when moving to the left. Initially, the moments on the left and the right sides of the skyrmion point to the left and to the right, respectively, as can be seen in Fig. 7(a). However, interaction with the dislocation introduces an asymmetry in the components of the magnetization. When the skyrmion is approaching the dislocation, Fig. 7(b), the red part of the skyrmion is facing the green end point of the dislocation. This creates a repulsive potential with two local magnetizations directions that are antiparallel and to lower the exchange interaction, these regions try to avoid each other. Since the STT current is pushing the skyrmion to the right in the figure, this external torque overcomes the repulsive potential and the skyrmion is moved along the upper side of the dislocation. The DM interaction coupled to the lattice defect causes a chirality and in the example shown in Fig. 7 favors a clockwise rotation of the magnetization. This pushes the skyrmion towards the upper part of the dislocation when it is moving to the right. In Fig. 7(d) the skyrmion is located at a position where it is bounded by ferromagnetic exchange interaction at both sides of the dislocation, causing a local minimum in the energy landscape. However, since the skyrmion has a finite linear momentum produced by the STT, it overcomes this bound state. As the skyrmion continues to move to the right along the dislocation it reaches the end point and here it stays for a short time on the right side of the dislocation. At this point the STT balances out the intrinsic exchange and DM interaction between the skyrmion and the lattice dislocation, thus, the right part of the skyrmion is pinned at the right side of the dislocation in another bound state, Fig. 7(e). The inertia of the skyrmion together with the clockwise rotation of the magnetization induced by the DM interaction now favors the skyrmion to move along the left direction under the dislocation. Notice also that the intensity of the spin current induced by the STT is not strong enough to overcome the inertia of the skyrmion during this short time. Figure 7(f) shows another possible bound state, similar to the one in Fig. 7(d), which again is overcome by the inertia of the skyrmion. Finally, in Fig. $7(\mathrm{~g})$ the skyrmion is pinned or trapped by the attractive potential between the green region of the dislocation and the green part of the skyrmion. On this way back to the final state, the skyrmion loses energy and linear momentum until it is trapped in the left side of the dislocation and, since the STT current is not sufficient to take the skyrmion out of this bounding potential, the skyrmion is pinned on the left side of the dislocation.

\section{CONCLUDING REMARKS}

In this paper, an efficient computational methodology, which enables a multiscale description of magnetization dynamics, and software are presented. The method proposed here is of the partitioned-domain type and is implemented in the UPPASD software. Using a number of examples, it is demonstrated that the continuum and the atomistic descriptions can be interfaced in a seamless way. As shown here, this enables simulations of magnetic phenomena at atomistic 
length scales, which are coupled to a micromagnetic description and which allow simulating cells that are of the same length scale as experimental samples.

As an illustration of the multiscale method, several examples of chiral and topological magnetic states are useddomain walls and skyrmions that interact with local perturbations of atomistic length scale. With this technique, an atomistic description of the Barkhausen effect is obtained. In addition, skyrmion dynamics is investigated. Of the more conspicuous results presented here, one may note the intricate dynamics of a skyrmion interacting with a lattice defect-an edge dislocation. The counterintuitive motion, which may go against an external STT, is analyzed in detail and is discussed in terms of the magnetic interactions coupled to the topology of the defect and the topology of the skyrmion.

The continuum regions have a certain discretization freedom compared to the atomistic region, which leads to the major advantage of the multiscale approach. However, as dynamics of objects, such as skyrmions and domain walls, is modelled, the mesh density of the continuum region should allow accurate resolution of the modelled objects. This would also mean that sometimes, if magnetization gradient is relatively large (i.e., fields change relatively rapidly), the continuum mesh must be relatively fine, which alleviates the computational advantages of using the continuum regions. One of the possible solutions in this case is using the dynamic remeshing and/or dynamically moving atomistic/continuum regions. This, however, might require additional resources and increases the complexity of the software.

The method proposed here allows simulating magnetic phenomena in general, e.g., in the field of magnonics or for studies of racetrack memories, with topological or chiral objects. Simulations of such phenomena calls for realistically sized simulation cells, which only a micromagnetic simulation model allows for. This level of description must be combined with an atomistic description, to treat regions where unavoidable lattice defects influence the magnetization dynamics, and the method outlined here allows for such a description opening up for simulations of many exciting magnetic phenomena and technologies.

The source code of the developed tool is freely available upon request to the authors (http://katalog.uu.se/empinfo/?id= N12-216).

\section{ACKNOWLEDGMENTS}

We are grateful for the support from STandUpp, eSSENCE, the Swedish Research Council, the Knut and Alice Wallenberg Foundation, the Foundation for Strategic Research and the Swedish Energy Agency (Energimyndigheten). We acknowledge D. Thonig for fruitful discussions.
[1] I. Žutić, J. Fabian, and S. Das Sarma, Spintronics: Fundamentals and applications, Rev. Mod. Phys. 76, 323 (2004).

[2] G. Burkard, H.-A. Engel, and D. Loss, Spintronics and quantum dots for quantum computing and quantum communication, Fortschr. Phys. 48, 965 (2000).

[3] E. Beaurepaire, J.-C. Merle, A. Daunois, and J.-Y. Bigot, Ultrafast Spin Dynamics in Ferromagnetic Nickel, Phys. Rev. Lett. 76, 4250 (1996).

[4] C. W. Abert, Discrete Mathematical Concepts in Micromagnetic Computations, PhD. thesis, Universitat Hamburg, 2013.

[5] O. Eriksson, A. Bergman, L. Bergqvist, and J. Hellsvik, Atomistic Spin-dynamics: Fundamentals and Applications (Oxford University Press, Oxford, 2017).

[6] C. J. Garcia-Cervera and A. M. Roma, Adaptive mesh refinement for micromagnetics simulations, IEEE Trans. Magn. 42, 1648 (2006).

[7] E. Kim and K. Lipnikov, The mimetic finite difference method for the landau-lifshitz equation, J. Comput. Phys. 328, 109 (2017).

[8] K. M. Tako, T. Schrefl, M. A. Wongsam, and R. W. Chantrell, Finite element micromagnetic simulations with adaptive mesh refinement, J. Appl. Phys. 81, 4082 (1997).

[9] R. F. L. Evans, W. J. Fan, P. Chureemart, T. A. Ostler, M. O. A. Ellis, and R. W. Chantrell, Atomistic spin model simulations of magnetic nanomaterials, J. Phys.: Condens. Matter 26, 103202 (2014).

[10] Q. Gong, M. Yi, and B.-X. Xu, Multiscale simulations toward calculating coercivity of Nd-Fe-B permanent magnets at high temperatures, Phys. Rev. Materials 3, 084406 (2019).
[11] Q. Gong, M. Yi, R. F. L. Evans, B.-X. Xu, and O. Gutfleisch, Calculating temperature-dependent properties of $\mathrm{Nd}_{2} \mathrm{Fe}_{14} \mathrm{~B}$ permanent magnets by atomistic spin model simulations, Phys. Rev. B 99, 214409 (2019).

[12] S. C. Westmoreland, R. F. L. Evans, G. Hrkac, T. Schrefl, G. T. Zimanyi, M. Winklhofer, N. Sakuma, M. Yano, A. Kato, T. Shoji, A. Manabe, M. Ito, and R. W. Chantrell, Multiscale model approaches to the design of advanced permanent magnets, Scr. Mater. 148, 56 (2018).

[13] F. Garcia-Sanchez, O. Chubykalo-Fesenko, O. Mryasov, R. W. Chantrell, and K. Y. Guslienko, Exchange spring structures and coercivity reduction in $\mathrm{FePt} / \mathrm{FeRh}$ bilayers: A comparison of multiscale and micromagnetic calculations, Appl. Phys. Lett. 87, 122501 (2005)

[14] T. Jourdan, A. Marty, and F. Lancon, Multiscale method for Heisenberg spin simulations, Phys. Rev. B 77, 224428 (2008).

[15] C. Andreas, A. Kákay, and R. Hertel, Multiscale and multimodel simulation of bloch-point dynamics, Phys. Rev. B 89, 134403 (2014).

[16] A. De Lucia, B. Krüger, O. A. Tretiakov, and M. Kläui, Multiscale model approach for magnetization dynamics simulations, Phys. Rev. B 94, 184415 (2016).

[17] R. Hertel, Applications of multi-scale modeling to spin dynamics in spintronics devices, in Handbook of Materials Modeling edited by W. Andreoni and S. Yip (Springer, Switzerland, 2018).

[18] B. Skubic, J. Hellsvik, L. Nordström, and O. Eriksson, A method for atomistic spin dynamics simulations: implementation and examples, J. Phys.: Condens. Matter 20, 315203 (2008). 
[19] UppASD uppsala university project website, http://physics.uu. se/uppasd.

[20] M. Poluektov, O. Eriksson, and G. Kreiss, Coupling atomistic and continuum modeling of magnetism, Comput. Methods Appl. Mech. Eng. 329, 219 (2018).

[21] M. Poluektov, O. Eriksson, and G. Kreiss, Scale transitions in magnetization dynamics, Commun. Comput. Phys. 20, 969 (2016).

[22] E. B. Tadmor and R. E. Miller, Modeling Materials (Cambridge University Press, Cambridge, UK, 2011).

[23] C. Ortner and L. Zhang, Energy-based atomistic-to-continuum coupling without ghost forces, Comput. Methods Appl. Mech. Eng. 279, 29 (2014).

[24] S. Qu, V. Shastry, W. A. Curtin, and R. E. Miller, A finitetemperature dynamic coupled atomistic/discrete dislocation method, Modell. Simul. Mater. Sci. Eng. 13, 1101 (2005).

[25] See Supplemental Material at http://link.aps.org/supplemental/ 10.1103/PhysRevResearch.2.013092 for technical aspects of the multiscale model, as well as materials parameters for the simulations presented in the main text. Videos illustrating the dynamics of Figs. 1,2,3-7 are also given here.

[26] A multidomain configuration is in general stabilized only if a dipole interaction is also included in the effective field. We omit this interaction in what follows and replace it by a magnetic structure prepared in a multidomain state. Since the domainwall structure is governed by the anisotropy and exchange interactions only, this step does not influence the shape or dynamics of the domain wall.

[27] A. Aharoni, Introduction to the Theory of Ferromagnetism, 2nd ed. (Oxford Science Publications, Oxford, 2000).

[28] O. A. Chubykalo, J. M. González, and J. González, Barkhausen jump distributions in a micromagnetic model, J. Magn. Magn. Mater. 184, L257 (1998).
[29] M. Pereiro, D. Yudin, J. Chico, C. Etz, O. Eriksson, and A. Bergman, Topological excitations in a kagome magnet, Nat. Commun. 5, 4815 (2014).

[30] K. Koumpouras, D. Yudin, C. Adelmann, A. Bergman, O. Eriksson, and M. Pereiro, A majority gate with chiral magnetic solitons, J. Phys.: Condens. Matter 30, 375801 (2018).

[31] W. Jiang, P. Upadhyaya, W. Zhang, G. Yu, M. B. Jungfleisch, F. Y. Fradin, J. E. Pearson, Y. Tserkovnyak, K. L. Wang, O. Heinonen, S. G. E. te Velthuis, and A. Hoffmann, Blowing magnetic skyrmion bubbles, Science 349, 283 (2015).

[32] S. Heinze, K. Von Bergmann, M. Menzel, J. Brede, A. Kubetzka, R. Wiesendanger, G. Bihlmayer, and S. Blügel, Spontaneous atomic-scale magnetic skyrmion lattice in two dimensions, Nat. Phys. 7, 713 (2011).

[33] It must be mentioned that since the process of skyrmion creation was the same in the atomistic and the continuum regions, within the latter, in the initial phase the skyrmion is not sufficiently continuous, but it rapidly evolves to a "smooth" solution (term "smooth" is not used in the strict mathematical sense here). This discrepancy could be the origin of the difference in temporal behavior. Therefore, if one is interested in the precise initial phase, an exclusively atomistic description must be used. However, at the final state, atomistic and continuum skyrmions are equivalent and both descriptions result in a breathing mode.

[34] S. Chikazumi and S. H. Charap, Physics of Magnetism (John Wiley \& Sons, New York, 1964).

[35] A. Fert, V. Cros, and J. Sampaio, Skyrmions on the track, Nat. Nanotechnol. 8, 152 (2013).

[36] J. Iwasaki, M. Mochizuki, and N. Nagaosa, Currentinduced skyrmion dynamics in constricted geometries, Nat. Nanotechnol. 8, 742 (2013). 